\title{
Results from the kaonic hydrogen X-ray measurement at DAFNE and outlook to future experiments
}

\author{
Michael Cargnelli* ; T. Ishiwatari, J. Marton, E. Widmann, J. Zmeskal \\ Stefan-Meyer-Institute for Subatomic Physics, Austrian Academy of Sciences, Vienna, Austria \\ michael.caronellidoeaw.ac.at
}

M. Bazzi, C. Berucci, A. Clozza, G. Corradi, C. Curceanu (Petrascu), A. d'Uffizi, C. Guaraldo, P. Levi Sandri, V. Lucherini, S. Okada, K. Piscicchia, M. Poli Lener, A. Rizzo, A. Romero Vidal, E. Sbardella, A. Scordo, H. Tatsuno, O. Vazquez Doce Laboratori Nazionali di Frascati dell'INFN, Frascati, Italy

G. Beer

Dep. of Phys. and Astro., Univ. of Victoria, Victoria B.C., Canada

L. Bombelli, C. Fiorini, R. Quaglia

Politechno di Milano, Sez. di Elettronica, Milano, Italy

A.M. Bragadireanu, M. Iliescu, D.L. Sirghi, F. Sirghi

Laboratori Nazionali di Frascati dell'INFN, Italy and IFIN-HH, Magurele, Bucharest, Romania

F. Ghio, B. Girolami

INFN Sez. di Roma I and Inst. Superiore di Sanita, Roma, Italy

R.S. Hayano, H. Shi

Univ. of Tokyo, Tokyo, Japan

M. Iwasaki

RIKEN, The Inst. of Phys. and Chem. Research, Saitama, Japan

P. Kienle

Tech. Univ. München, Physik Dep., Garching, Germany

D. Pietreanu, T. Ponta, A. Tudorache, V. Tudorache 


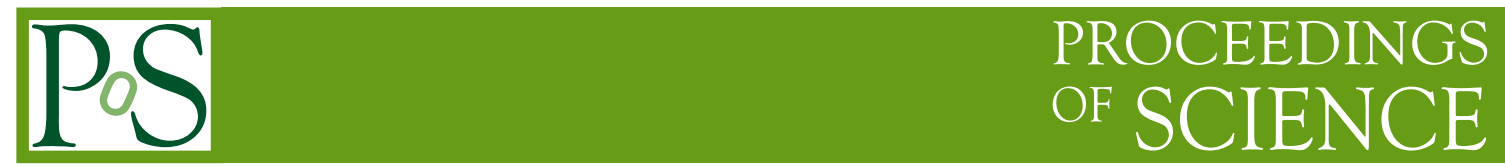

IFIN-HH, Magurele, Bucharest, Romania

The $\bar{K} N$ system at rest plays a key role for understanding the strong interaction of hadrons with strangeness involved. The experiment SIDDHARTA used X-ray spectroscopy of kaonic atoms to measure the strong-interaction-induced shift and width of the ground state. It was the first experiment on kaonic He-3 and deuterium and kaonic hydrogen was measured with improved precision resulting in $\varepsilon_{1 s}=-283 \pm 36$ (stat) \pm 6 (syst) $\mathrm{eV}$ and $\Gamma_{1 s}=541 \pm 89$ (stat) \pm 22 (syst) eV. Additionally a plan for a future quantitative experiment on kaonic deuterium is introduced in this contribution.

8th International Conference on Nuclear Physics at Storage Rings-Stori11,

October 9-14, 2011

Laboratori Nazionali di Frascati dell'INFN, Italy

\footnotetext{
${ }^{*}$ Speaker.

$\dagger$ We Acknowledge the support of the European Community-Research Infrastructure Integrating Activity "Study of Strongly Interacting Matter" (acronym HadronPhysics2, Grant Agreement no. 227431) under the Seventh Framework Programme of EU; HadronPhysics I3 FP6 European Community program, Contract No. RII3-CT-2004-506078; Austrian Federal Ministry of Science and Research BMBWK 650962/0001 VI/2/2009; Romanian National Authority for Scientific Research, Contract No. 2-CeX 06-11-11/2006; and the Grant-in-Aid for Specially Promoted Research (20002003), MEXT, Japan.
} 


\section{Introduction}

The strong interaction between hadrons at low energies can not be described in terms of quarks and gluons. Instead, effective field theories are used which rely on experimental input. The data come from kaon scattering experiments, atomic X-ray measurements and the energy and width of known resonances.

The kaonic atom experiments measure the effect of the strong interaction on the X-ray transitions, namely the shift and width of the ground state. These quantities are key input for theory and permit testing the ability of theoretical models to accommodate the different types of experimental information. For current theory see [U, []] and references therein.

The $\bar{K} N$ interaction at rest is normally described in terms of complex scattering lengths. To extract the isospin dependant $\bar{K} N$ scattering lengths $a_{0}$ and $a_{1}$, a first measurement of kaonic deuterium is necessary in addition to kaonic hydrogen.

Kaonic atoms are bound electromagnetically, the energy levels with strong interaction "switched off" are known precisely from QED. The strong interaction modifies the binding and causes the absorption of the kaon by the nucleus. This leads to a significant shift and broadening of the $1 s$ state of kaonic $\mathrm{Z}=1$ atoms, higher states are only marginally affected. By measuring the $\mathrm{K}$-series $\mathrm{X}$-ray transitions the strong-interaction induced shift and width are directly observable. Via Deser-type formulae [团] the shift and width are connected with the real and imaginary part of the $K^{-} \mathrm{p}$ and $K^{-} \mathrm{d}$ scattering length, which themselves are linear combinations of the isospin scattering lengths.

The previous experimental results for kaonic hydrogen (KpX [可] and DEAR [四]) suffer from limited precision, and the improvement reported herein is of high relevance for testing and advancing the theory in this field. Furthermore, by using only DEAR results, most of the theoretical models had difficulties in explaining all experimental evidence in a consistent way [B]].

\section{Experiment}

Fig. 1 illustrates the principle of our experiment: Both kaons of a charged kaon pair are detected and define the trigger. Hits in the X-ray detectors coincident with the trigger contain kaonic hydrogen signals along with background from kaon decay and kaon absorption in hydrogen and structure materials. Due to the limited timing resolution, some accidental (not kaon-correlated) background remains.

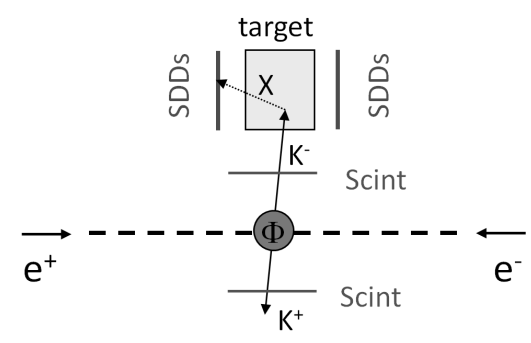

Figure 1: Schematic view of the SIDDHARTA setup at DAФNE. 
The $e^{+} e^{-}$collider DA $\Phi$ NE of Laboratori Nazionali di Frascati produced a peak luminosity of $310^{32} \mathrm{~cm}^{-2} \mathrm{~s}^{-1}$ and an integrated usable ${ }^{1}$ luminosity of up to $8 \mathrm{pb}^{-1}$ per day. The 2.2 liter cryogenic gas target of was operated at $1.5-1.8 \%$ of liquid hydrogen density. The entrance window was $20 \mathrm{~cm}$ above the interaction point (IP). The kaon trigger consisted of two $1.5 \mathrm{~mm}$ thick plastic scintillators situated $6 \mathrm{~cm}$ above and below the IP.

A kaonic hydrogen atom is formed when a negative kaon enters the target cell, looses its remaining kinetic energy through ionization and excitation and finally is captured, replacing the electron, in an excited orbit $(\mathrm{n} \simeq 25)$. Via different cascade processes the kaonic atom deexcites to lower states. When the kaon reaches a low-n state with small angular momentum, it is absorbed by the nucleus. A small fraction of kaonic hydrogen atoms survive long enough to make one of the (np-1s) transitions (K-series).

The experimental challenge is the very small kaonic hydrogen X-ray yield, and the difficulty in doing X-ray spectroscopy in the radiation environment of an accelerator. Therefore new X-ray detectors [ [ $]$ were developed and built in the framework of the SIDDHARTA ${ }^{2}$ project.

For each event, Tthe data acquisition system stored the signal amplitudes of hits on the 144 SDDs and, if a kaontrigger was present, the time correlation between X-ray and kaon.

The basic energy calibration for each of the SDDs was obtained from periodic measurements of fluorescence lines from $\mathrm{Ti}$ and $\mathrm{Cu}$ foils excited by an $\mathrm{X}$-ray tube during beam. This procedure delivered the information to compensate the individual gains, making it possible to sum the 144 spectra. The response function of the overlayed detector-system was found to deviate slightly from the pure Gaussian shape (Fig. 2). Various approximations to account for this effect were tested, e.g. 2 exponential tail-functions convoluted in each fit evaluation. The rate dependance was studied and finally the parameters of the response width were derived from calibration data taken with very low X-ray tube current, which resulted in SDD rates similar to those in real data taking.

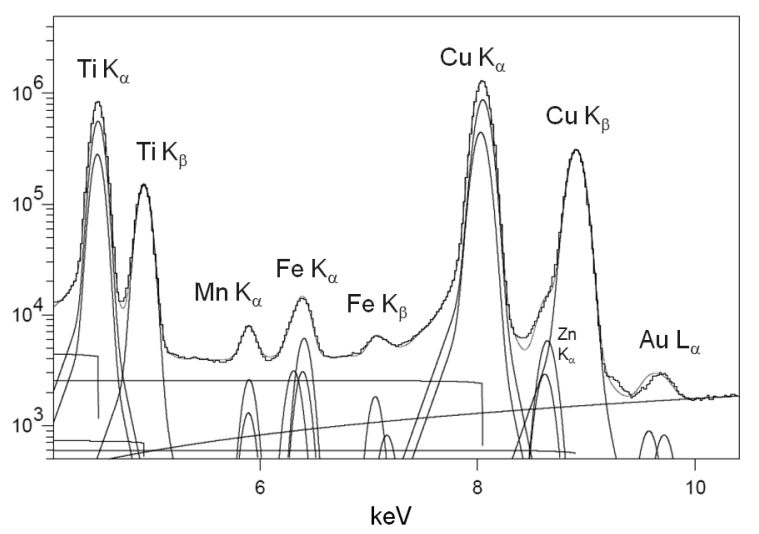

Figure 2: Calibration X-ray spectrum originated by irradiation of foils in the setup by an external X-ray tube. Data taken with beam on. The spectrum shown is the sum of 100 spectra from individual gain-aligned detectors. The $K_{\alpha}$ lines consist of $K_{\alpha 1}$ and $K_{\alpha 2}$.

Using the $\mathrm{Cu}$ and $\mathrm{Au}$ fluorescence lines in the untriggered spectra with $\mathrm{X}$-ray tube off, but excited by background, we derived a small gain correction of a few $\mathrm{eV}$ which results from rate

\footnotetext{
${ }^{1}$ During injection of $e^{+}$and $e^{-}$into the rings, high beam-background prohibited X-ray measurements

${ }^{2}$ Silicon Drift Detectors for Hadronic Atom Research by Timing Application
} 
difference between data with and without the X-ray tube. Additionally we checked the calibration using the triggered spectra and the kaonic lines from wallstops.

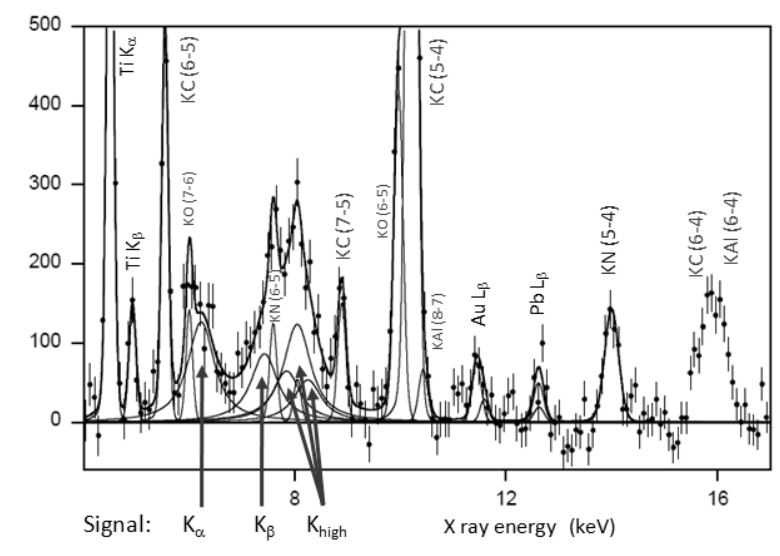

Figure 3: X-ray spectrum of kaonic hydrogen measured in SIDDHARTA. The continuous background fitcomponent is already subtracted. The kaonic lines are from stops in the kapton foils of the target walls. Their intensities are from a combined fit with the deuterium spectrum, in which they are clearly distinguishable.

For the fit the kaonic hydrogen K-series transitions were represented by Voigt functions with the (energy dependant) Gauss sigma fixed to the values obtained by interpolating the values from the calibration lines. The pattern of the transition energies is given by the electro-magnetic values. The background lines (kaonic carbon etc. from stops in foils of the target windows) were Gaussians, the continuous background, a quadratic polynomial. The total spectrum was convoluted with the tail functions ${ }^{3}$. Since the individual lines from the higher transitions are high in total intensity, but completely overlapped, they tend to spoil the determination of shift and width of the K-series and a fixing of the intensity ratio would introduce a model-dependance. Therefore we concentrated our analysis on the $K_{\alpha}$ and $K_{\beta}$ lines while the $K_{\text {high }}$ lines were treated as "background". The procedure is as follows: start with a fit with all line intensities free, free common shift of all transitions, one Lorentz width for all transitions. Then fix the line position of the (4-1) and higher transitions, fit free common shift of $K_{\alpha}, K_{\beta}$. Modify the fixed line positions by the last shift result then resume at the previous step. After few iterations we obtained a consistent shift and width for all transitions. The result was:

$$
\varepsilon_{1 s}=-283 \pm 36 \text { (stat) } \pm 6 \text { (syst) } \mathrm{eV} \text { and } \Gamma_{1 s}=541 \pm 89 \text { (stat) } \pm 22 \text { (syst) } \mathrm{eV} .
$$
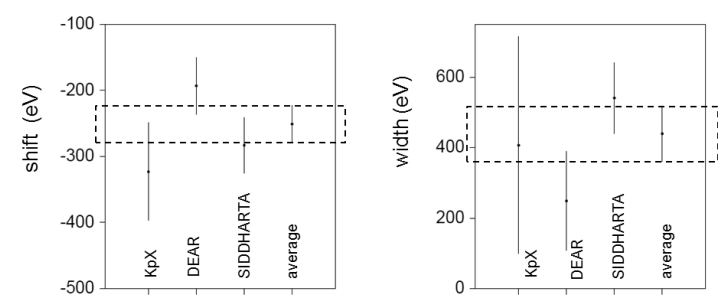

Figure 4: Compilation of experimental results for the ground-state shift and width of kaonic hydrogen. The statistic and systematic errors are added linearly.

\footnotetext{
${ }^{3}$ various models were tested, their deviation contributed to the systematic error
} 


\section{Towards SIDDHARTA-2}

The analysis of the kaonic deuterium (KD) data (see Fig. 5) in SIDDHARTA showed a 2 sigma hint on the signal, establishing only an upper limit for the K-series yield. A quantitative measurement of KD X-rays - determining shift and width - requires a substantial improvement of the applied experimental technique. Since the yield is expected to be about $1 / 10$ of the KH yield and the width about twice that of $\mathrm{KH}$, we need a reduction in background of about 20 and an increase of detection efficiency of 2-3 along with an integrated luminosity of around $800 \mathrm{pb}^{-1}$. Given such a situation, our Monte Carlo studies approximate a reachable precision of about $70 \mathrm{eV}$ for the shift and $160 \mathrm{eV}$ for the width.

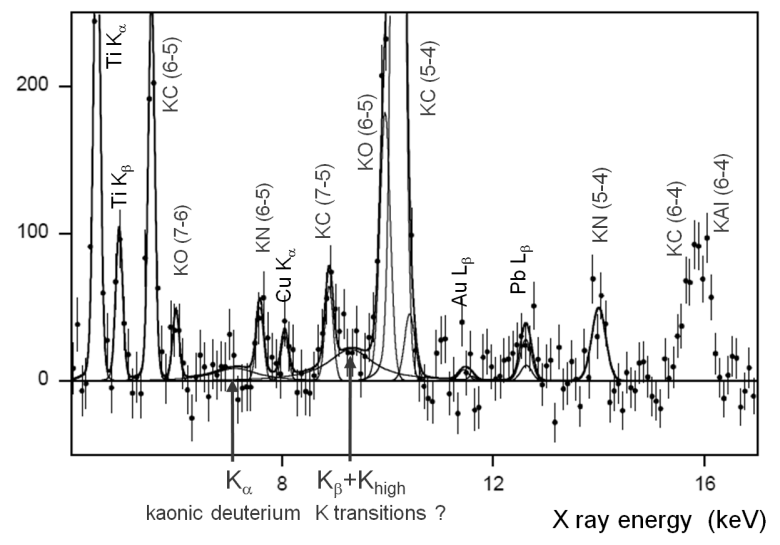

Figure 5: Kaonic deuterium. The continuous background fit-component is already subtracted. Fit with fixed KD transition shift and width $(-800,1000 \mathrm{eV})$.

There are four changes proposed for the experimental technique:

(1) Trigger geometry and target density: By placing the upper kaon-trigger detector closely in front of the target entry window, the probability that a triggered kaon really enters the gas and is stopped there is much enlarged. Making the detector smaller than the entry area gives away some signal, but suppresses efficiently the kaonic lines from "wallstops" (kaons entering the gas volume, but passing from inside to the cylindrical target walls). The "signal per trigger" number goes up, which also reduces the accidental background coming along with every trigger. We intend to double the gas density which enhances the gasstops and further reduces the wallstops.

(2) $\mathrm{K}^{+}$discrimination by 2 complementary methods: A "kaonstopper" scintillator is placed directly below the lower kaon trigger. When a $K^{-}$stops there, only one (large) signal from pileup of stopping and kaon-absorption secondaries is seen, however in the case of a $K^{+}$, the kaon-decay particles are seen after the signal from the stopping (mean $K^{+}$lifetime $12.8 \mathrm{~ns}$ ), with a flash-ADC we will be able to distinguish the 2 cases with high efficiency. In addition we will use scintillators surrounding the target to measure $K^{-}$absorption secondaries (pions). The time-window for gasstops is about $4 \mathrm{~ns}$ wide. By this condition we also suppress stops in the entry window.

(3) Active shielding: The scintillators surrounding the target will also be used as a prompt anticoincidence if the spatial correlation of SDDs hit and scintillator indicates that the hit has originated from a pion ("charged particle veto"). An anticoincidence covering the SDD time window of about $600 \mathrm{~ns}$ (with an exception of the $4 \mathrm{~ns}$ of the stopping time in the gas) will reduce the acciden- 
tal background. Although the scintillators have only low efficiency for gammas, the high rate of secondaries from the electromagnetic showers allows a relevant reduction of accidental ("beam") background. Remarkably, the upper trigger scintillator has 2 functions, it is also used as an anticoincidence counter: after the passing of the kaon and eventual prompt kaon-absorption secondaries, it vetos beam-background.

(4) Operate SDDs at lower temperature: tests indicate that an improvement of the timing resolution by a factor of 1.5 is feasible by more cooling. The energy resolution decreases somewhat, but will still be sufficient for the broad KD transitions.

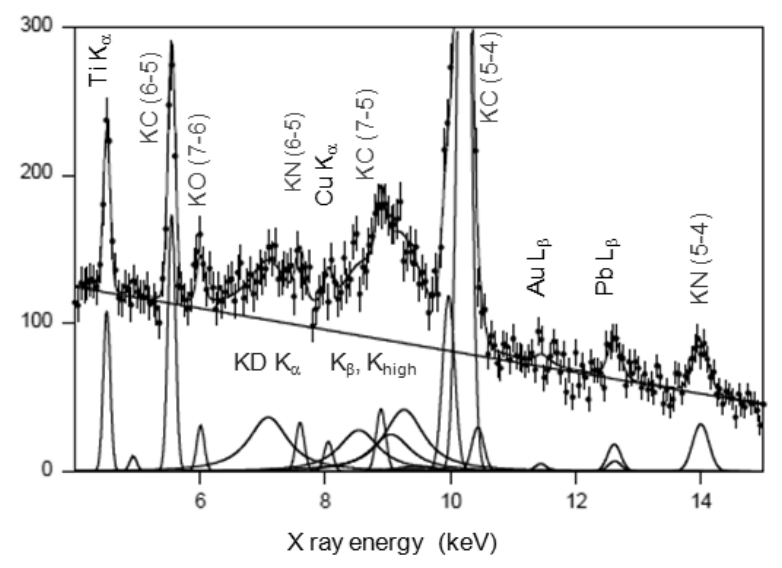

Figure 6: Monte Carlo simulation of the kaonic deuterium spectrum corresponding to the proposed SIDDHARTA-2 techniques.

The signal enhancement is due to moving the target cell closer to the IP, by changing its shape, by better solid angle of the SDDs and by the higher gas-density.

\section{Summary and Conclusion}

Within the SIDDHARTA project we measured X-ray transitions of light kaonic atoms [Q, एव, [D] The experimental part covering kaonic hydrogen $(\mathrm{KH})$ delivered the most precise measurement of the $K$-series X-rays in $\mathrm{KH}$ [[]]. Our determination of the shift and width provides new constraints on theories, having reached a quality which will demand refined calculations of the low-energy $\bar{K} N$ interaction.

We have now worked out an improved experimental technique [[2] which, using the existing SDD detectors, promises to allow a measurement of the shift and width in kaonic deuterium, long awaited data for the description of the strong interaction at lowest energies in hadronic systems with strangeness.

\section{References}

[1] M. Döring, U.-G. Meißner, Phys. Lett. B 704663 (2011)

[2] Y. Ikeda, T. Hyodo, W. Weise, Phys. Lett. B 70663 (2011)

[3] W. Weise, Nucl. Phys. A 83551 (2010) 
[4] U.-G. Meißner, U. Raha, A. Rusetsky, Eur. Phys. J. C 35349 (2004)

[5] M. Iwasaki, et al. Phys. Rev. Lett. 783067 (1997)

[6] G. Beer, et al. DEAR Collaboration, Phys. Rev. Lett. 94212303 (2005)

[7] M. Bazzi, et al., Nucl. Instrum. Methods Phys. Res. A 628264 (2011)

[8] M. Bazzi, et al. SIDDHARTA Collaboration, Phys. Lett. B 704113 (2011)

[9] M. Bazzi, et al. SIDDHARTA Collaboration, Phys. Lett. B 693199 (2011)

[10] M. Bazzi, et al. SIDDHARTA Collaboration, Phys. Lett. B 681310 (2009)

[11] D.L. Sirghi, et al. In this proceedings

[12] SIDDHARTA-2 Collaboration, Proposal of Laboratori Nazionali di Frascati of INFN, The upgrade of the SIDDHARTA apparatus for an enriched scientific case (2010) 